\title{
Characterization of trap states in perovskite films by simultaneous fitting of steady- state and transient photoluminescence measurements
}

Cite as: J. Appl. Phys. 124, 073102 (2018); https://doi.org/10.1063/1.5029278

Submitted: 12 March 2018 . Accepted: 20 July 2018 . Published Online: 16 August 2018

Xiao Fu, Klaus J. Weber, and Thomas P. White (D)
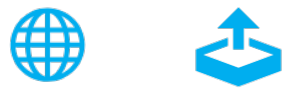

\section{ARTICLES YOU MAY BE INTERESTED IN}

Unusual defect physics in $\mathrm{CH}_{3} \mathrm{NH}_{3} \mathrm{~Pb} / 3$ perovskite solar cell absorber

Applied Physics Letters 104, 063903 (2014); https://doi.org/10.1063/1.4864778

Dependence of power conversion properties of perovskite solar cells on operating temperature

Applied Physics Letters 113, 113501 (2018); https://doi.org/10.1063/1.5041028

Research Update: Recombination and open-circuit voltage in lead-halide perovskites

APL Materials 6, 100702 (2018); https://doi.org/10.1063/1.5052164

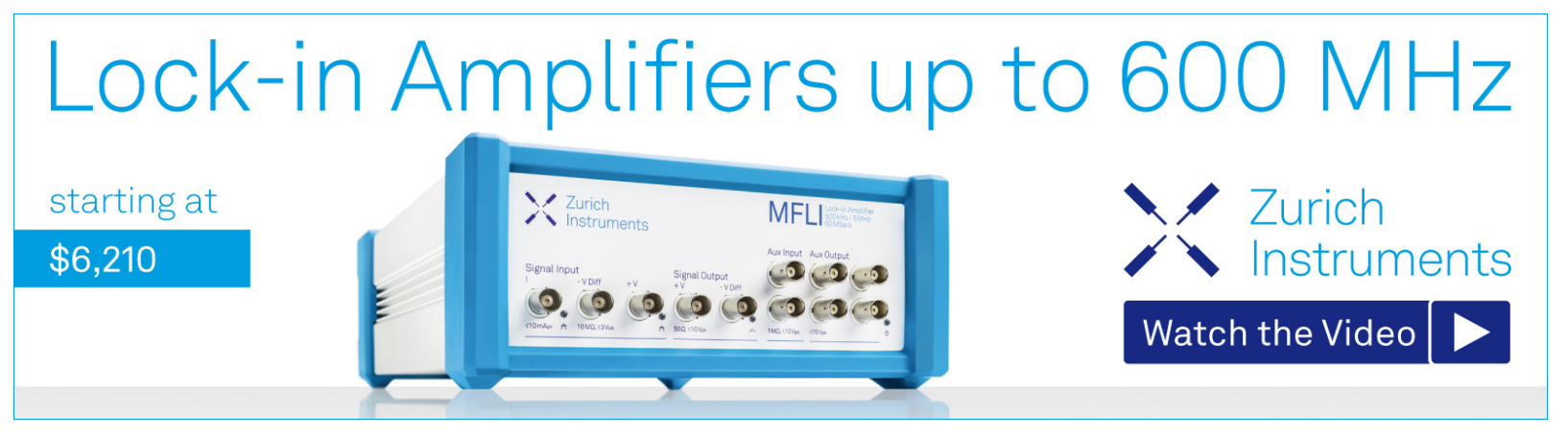




\title{
Characterization of trap states in perovskite films by simultaneous fitting of steady-state and transient photoluminescence measurements
}

\author{
Xiao Fu, Klaus J. Weber, and Thomas P. White \\ Research School of Engineering, The Australian National University, Canberra 2601, Australia
}

(Received 12 March 2018; accepted 20 July 2018; published online 16 August 2018)

\begin{abstract}
Understanding carrier recombination mechanisms and quantifying recombination dynamics are key to improving the performance of state-of-the-art perovskite solar cells. Here, we present a method to quantify the quality of perovskite thin films using a combination of steady-state and transient photoluminescence measurements. The combined experimental datasets are fitted using a single, general recombination model, from which detailed trap and recombination parameters can be extracted, and the accuracy of the fitted values is estimated. This approach expands the application of photoluminescence measurements to include quantitative evaluation of the most relevant defect characteristics, including trap density, energy level, and carrier capture coefficients. We apply this approach to compare perovskite films of the widely studied methyl-ammonium lead iodide $\left(\mathrm{MAPbI}_{3}\right)$ with the high performance quadruple-cation, mixed-halide composition $\mathrm{Cs}_{0.07} \mathrm{Rb}_{0.03}\left(\mathrm{FA}_{0.85} \mathrm{MA}_{0.15}\right)_{0.9} \mathrm{~Pb}\left(\mathrm{I}_{0.85} \mathrm{Br}_{0.15}\right)_{3}$. Our quantitative analysis of trap properties in these perovskite films suggests that the superior performance of the quadruple cation films may be due to a greatly reduced electron capture coefficient, rather than a significant reduction in the trap density. Published by AIP Publishing. https://doi.org/10.1063/1.5029278
\end{abstract}

\section{INTRODUCTION}

Hybrid organic-inorganic perovskite materials with the chemical composition $\mathrm{ABX}_{3}\left[\mathrm{~A}=\mathrm{Cs}^{+}, \mathrm{Rb}^{+}, \mathrm{CH}_{3} \mathrm{NH}_{3}{ }^{+}\right.$ (methylammonium cations or $\mathrm{MA}$ ), or $\mathrm{CH}\left(\mathrm{NH}_{2}\right)_{2}{ }^{+}$(formamidinium cations or $\mathrm{FA}$ ); $\mathrm{B}=\mathrm{Pb}^{2+}$ or $\mathrm{Sn}^{2+}$; and $\mathrm{X}=\mathrm{I}^{-}$, $\mathrm{Br}^{-}$, or $\mathrm{Cl}^{-}$] were first applied to solar cells in 2009. ${ }^{1}$ Since then, the performance of hybrid lead halide perovskite solar cells has improved dramatically, leading to the current efficiency record of $22.7 \% .^{2}$ The rapid emergence of this technology has been driven by developments in several areas including cell structures, deposition methods, material compositions, and improved understanding of perovskite material properties.

One of the defining characteristics of perovskite solar cells is their high open circuit voltage, with the best mixedcation, mixed-halide compositions achieving a $V_{O C}$ of $1.2 \mathrm{~V}$ with a bandgap of $\sim 1.6 \mathrm{eV} .^{3}$ Such performance results from the excellent optoelectronic properties of solution-processed perovskite films and, in particular, their high radiative efficiency. In two recent reviews, Tress ${ }^{4}$ and Stranks ${ }^{5}$ discussed the importance of radiative efficiency for cell performance and identified further reductions in non-radiative recombination as the key to boosting cell efficiency toward theoretical limits.

Under normal operating conditions, non-radiative recombination in perovskite solar cells is dominated by trap-assisted recombination, also referred to as Shockley-Read-Hall (SRH) recombination. ${ }^{6}$ The physical and chemical origin of such traps (or defects) in perovskite materials is an ongoing research topic, but intrinsic defects such as lattice vacancies and interstitial ionic species have been identified as likely contributors. Experimental characterization of recombination dynamics and trap properties is an essential step toward identifying and eventually mitigating specific trap states and requires accurate and reliable methods for comparing different samples and material compositions.

The three main physical parameters that determine how a particular trap state will impact the non-radiative recombination rate are the trap density $\left(N_{t}\right)$, energy level $\left(E_{t}\right)$, and capture cross sections for electrons and holes $\left(\sigma_{n}\right.$ and $\sigma_{p}$, respectively). ${ }^{4}$ Measuring these parameters directly is challenging, and few techniques allow all parameters to be extracted simultaneously. Electrical characterization methods for measuring the trap density and energy level include thermally stimulated current and capacitance vs. frequency measurements at different temperatures. ${ }^{7-10}$ However, both techniques require electrical contacts on the sample which can introduce additional recombination, while the application of an electrical bias can also induce ion migration. Temperature-dependent measurements also present challenges for perovskites due to the possibility of phase transitions and/or thermal degradation.

Steady-state and transient photoluminescence measurements are widely used for studying carrier recombination in perovskite films and solar cells as they are fast and noncontact and can be applied to films, partial devices, and complete cells. Both types of measurements are commonly used to compare the relative quality of samples, where high PL intensity and/or long lifetimes are typically associated with improved cell performance. Alternatively, more detailed quantitative data can be extracted by fitting the experimental measurements to a theoretical recombination model. Ideally, such a model should not only provide a good fit to the experimental data but also allow relevant physical parameters to be extracted.

The most common recombination models used to fit transient measurements are based on the simple rate equation $\frac{d n}{d t}=G-k_{1} n-k_{2} n^{2}-k_{3} n^{3}$, where $n$ is the electron density, 
$G$ is the charge-carrier generation rate, $k_{1}$ is the trap-assisted recombination rate, $k_{2}$ is the radiative recombination rate, and $k_{3}$ is the Auger recombination rate. ${ }^{11,12}$ A major limitation of this approach is that the trap-assisted recombination is described by a single rate constant $k_{2}$, which does not directly relate to the relevant physical trap parameters (density, energy level, and cross-section) identified above. Furthermore, the rate equation is strictly only valid when the electron density $(n)$ and hole density $(p)$ are equal and when the concentration of generated carriers is much higher than the intrinsic carrier concentration $\left(n_{i}\right)$. Only under these conditions, the radiative recombination rate is proportional to $n^{2}$; more generally, it scales as $\left(n p-n_{i}^{2}\right)$.

The simple rate equation given above was extended by Stranks and Manger ${ }^{5,13}$ to include separate terms for capture and emission of free carriers, as well as the dependence on the trap density and capture cross section. These works provided new insights into carrier recombination dynamics, but the trap energy level was still not explicitly included in the model.

A complete physical model of SRH recombination including all three physical trap parameters was used by Wen et al. ${ }^{14}$ to fit steady-state PL measurements as a function of the excitation level. However, as we discuss below, steady-state PL measurements depend only weakly on the trap energy level and capture cross-section, and thus, the accuracy with which these parameters can be estimated with this approach is relatively low.

To overcome the limitations of the above methods, here we apply a single, general transient recombination model to fit simultaneously both steady-state and transient PL measurements as a function of excitation intensity. With this approach, we are able to extract recombination coefficients, trap density, trap energy level, capture cross section, and doping density and to estimate the accuracy of the fitted parameters using a sensitivity analysis. The technique is demonstrated on both $\mathrm{CH}_{3} \mathrm{NH}_{3} \mathrm{PbI}_{3}$ and high-performance quadruple-cation $\mathrm{Cs}_{0.07} \mathrm{Rb}_{0.03}\left(\mathrm{FA}_{0.85} \mathrm{MA}_{0.15}\right)_{0.9} \mathrm{~Pb}\left(\mathrm{I}_{0.85} \mathrm{Br}_{0.15}\right)_{3}$ perovskite films, providing a direct comparison of differences in the trap properties in these two perovskite compositions. Interestingly, the most notable difference between the two film compositions is seen in the hole capture cross-section, which differ by two orders of magnitude. While further study is required to verify these observations, the results may provide new insights into the origin of the improved performance of mixed-cation/ mixed-halide perovskites. These results demonstrate the utility of photoluminescence analysis for quantitatively studying trap states in perovskite films.

\section{THEORY}

In 1952, Shockley and Read investigated the FermiDirac statistics of the recombination of electrons and holes through traps. ${ }^{15}$ In the original paper, the theory of trapassisted recombination kinetics in non-degenerate semiconductors was established in agreement with the experimental results obtained by Hall. ${ }^{16,17}$ Here, we follow the original theory to model the dynamics of carriers and traps during transient and steady-state PL measurements. Table I lists the symbols used in the analytical solution, and the equations related to SRH recombination [Eqs. (5)-(7)] were derived by Shockley and Read.

The density of electrons and holes follows the differential equations

$$
\begin{aligned}
& \frac{d n}{d t}=G-\left(U_{\text {rad }}+U_{S R H_{n}}+U_{\text {Aug }}\right), \\
& \frac{d p}{d t}=G-\left(U_{\text {rad }}+U_{S R H_{p}}+U_{\text {Aug }}\right),
\end{aligned}
$$

where the radiative recombination rate $\left(U_{\text {rad }}\right)^{18}$ and the Auger recombination rate $\left(U_{A u g}\right)^{19}$ are given by

$$
\begin{gathered}
U_{\text {rad }}=B\left(n p-n_{i}^{2}\right), \\
U_{\text {Aug }}=\Gamma_{n} n\left(n p-n_{i}^{2}\right)+\Gamma_{p} p\left(n p-n_{i}^{2}\right) .
\end{gathered}
$$

Under steady-state conditions, the net rate capture of electrons must be equal to that of holes $\left(U_{S R H_{n}}=U_{S R H_{p}}\right)$. Thus, the net rate of SRH recombination can be expressed as

$$
U_{S R H}=\frac{C_{n} C_{p}\left(n p-n_{1} p_{1}\right)}{C_{n}\left(n+n_{1}\right)+C_{p}\left(p+p_{1}\right)} .
$$

However, the SRH recombination rates of electrons and holes $\left(U_{S R H_{n}}\right.$ and $\left.U_{S R H_{p}}\right)$ are not necessarily equal during transient PL measurements. Therefore, in the general case, the rate of electrons being trapped by the defect state $U_{S R H_{n}}$ and the rate of the trapped electrons being emitted from the defect state and relaxing to the valence band $U_{S R H_{p}}$ are expressed, respectively, by

$$
\begin{gathered}
U_{S R H_{n}}=C_{n}\left[\left(1-f_{t}\right) \Delta n-\left(n_{0}+n_{1}\right) \Delta f_{t}\right], \\
U_{S R H_{p}}=C_{p}\left[f_{t} \Delta p+\left(p_{0}+p_{1}\right) \Delta f_{t}\right] .
\end{gathered}
$$

The initial value $f_{t 0}=\frac{1}{1+n_{1} / n_{0}}=1-\frac{1}{1+p_{1} / p_{0}}$ refers to equilibrium conditions, $f_{t}=f_{t 0}+\Delta f_{t}$, and electrical neutrality requires that $\Delta p-\Delta n=N_{t} \Delta f_{t}$. Thus, the change in the fraction of occupied traps depends on the trap density. Meanwhile, $C_{n}$ and $C_{p}$ will be affected by both the trap density and the capture cross section of electrons and holes, where $C_{n}=N_{t}\left\langle c_{n}\right\rangle=N_{t} \sigma_{n} v_{t h}$ and $C_{p}=N_{t}\left\langle e_{n}\right\rangle=N_{t} \sigma_{p} v_{t h}$. Also, $n_{1}=N_{C} \exp \left(\frac{E_{t}-E_{C}}{k T}\right)$ and $p_{1}=N_{V} \exp \left(\frac{E_{V}-E_{t}}{k T}\right)$ have a strong dependence on the trap energy level. Therefore, apart from constants, all variables used in the equations of SRH recombination can be expressed by the trap density $\left(N_{t}\right)$, trap energy level $\left(E_{t}\right)$, and the capture cross sections of electrons and holes $\left(\sigma_{n}\right.$ and $\left.\sigma_{p}\right)$.

For the required constants in the model, the semiconductor bandgap $E_{g}$ was extracted from measured PL spectra (Fig. S2 in the supplementary material). The effective densities of states $N_{C}$ and $N_{V}$ of the perovskite materials are calculated according to the reported effective masses of electrons and holes $\left(m_{e}^{*}\right.$ and $\left.m_{h}^{*}\right) .{ }^{20}$ Furthermore, following the approach of Stranks, Manger, and Wen,,$^{5,13,14}$ we assume that the recombination is dominated by a single type of trap, although the presence of multiple traps has been reported. ${ }^{8,10,21}$ This is sufficient to obtain very good fits to 
TABLE I. Symbols.

\begin{tabular}{lc}
\hline \hline$n\left(\mathrm{~cm}^{-3}\right)$ & Electron density in the conduction band \\
$p\left(\mathrm{~cm}^{-3}\right)$ & Hole density in the valence band \\
$G\left(\mathrm{~cm}^{-3} \mathrm{~s}^{-1}\right)$ & Generation rate \\
$U_{\text {rad }}\left(\mathrm{cm}^{-3} \mathrm{~s}^{-1}\right)$ & Radiative recombination rate \\
$U_{S R H_{n}}\left(\mathrm{~cm}^{-3} \mathrm{~s}^{-1}\right)$ & Shockley-Read-Hall recombination rate of electrons \\
$U_{S R H_{p}}\left(\mathrm{~cm}^{-3} \mathrm{~s}^{-1}\right)$ & Shockley-Read-Hall recombination rate of holes \\
$U_{A u g}\left(\mathrm{~cm}^{-3} \mathrm{~s}^{-1}\right)$ & Auger recombination rate \\
$B\left(\mathrm{~cm}^{3} \mathrm{~s}^{-1}\right)$ & Radiative recombination coefficient \\
$n_{i}\left(\mathrm{~cm}^{-3}\right)$ & Intrinsic carrier density \\
$\Gamma_{n}\left(\mathrm{~cm}^{6} \mathrm{~s}^{-1}\right)$ & Auger recombination coefficient of electrons \\
$\Gamma_{p}\left(\mathrm{~cm}^{6} \mathrm{~s}^{-1}\right)$ & Auger recombination coefficient of holes \\
$C_{n}\left(\mathrm{~s}^{-1}\right)$ & Shockley-Read-Hall recombination coefficient of electrons \\
$C_{p}\left(\mathrm{~s}^{-1}\right)$ & Fraction of traps occupied by electrons \\
$f_{t}$ & Trap density \\
$N_{t}\left(\mathrm{~cm}^{-3}\right)$ & Trap energy level (below conduction band) \\
$E_{t}(\mathrm{eV})$ & The number of electrons in the conduction band \\
$n_{1}\left(\mathrm{~cm}^{-3}\right)$ & for the case in which the Fermi level falls at $E_{t}$ \\
$p_{1}\left(\mathrm{~cm}^{-3}\right)$ & The number of holes in the valence band for \\
$\left\langle c_{n}\right\rangle\left(\mathrm{cm}^{3} \mathrm{~s}^{-1}\right)$ & the case in which the Fermi level falls at $E_{t}$ \\
$\left\langle e_{n}\right\rangle\left(\mathrm{cm}^{3} \mathrm{~s}^{-1}\right)$ & Capture coefficient of electrons \\
$\sigma_{n}\left(\mathrm{~cm}^{2}\right)$ & Emission coefficient of electrons \\
$\sigma_{p}\left(\mathrm{~cm}^{2}\right)$ & (or capture coefficient of holes) \\
$v_{t h}\left(\mathrm{~cm}^{2}\right)$ & Capture cross section of electrons \\
$N_{A}\left(\mathrm{~cm}^{-3}\right)$ & Capture cross section of holes \\
\hline \hline & Thermal velocity \\
\hline & Acceptor density
\end{tabular}

the experimental parameters, suggesting either that the assumption of a single trap state is valid or that the extracted trap parameters correspond to "effective" values with contributions from several different trap states. It is not possible to separate these two possibilities with the available data.

\section{NUMERICAL IMPLEMENTATION}

The recombination expressions summarized in the section on "Theory" were implemented in a time-domain numerical model that could be used to simulate both steadystate and transient experimental conditions. To reproduce a steady-state PL intensity measurement in the model, a constant generation term was set at time $t=0$, and the time-dependent recombination rates [Eqs. (6) and (7)] were calculated until they reached equilibrium where the SRH recombination rates of electrons and holes were equal [Fig. 1(a)]. Intensity-dependent PL intensity curves were produced by repeating this calculation as a function of the generation rate. Examples of such curves are shown in Figs. 2(a), 2(c), and 2(e), calculated for representative parameter values. When fitting the model to the experimental data, it was necessary to scale the modelled data by a constant value as the experimental measurements were uncalibrated and included optical collection losses. Experimental generation rates were calculated from the laser wavelength, the laser intensity, and the absorption coefficient. The detailed experimental settings are described in the supplementary material.

To simulate a transient PL measurement, we approximated the $\sim 110$ ps excitation laser pulse by a square generation profile and calculated the transient radiative recombination rates following the pulse. The results of such a simulation are shown in Fig. 1(b). The normalized decay curve on the right-hand side of Fig. 1(b) is equivalent to the measured PL decay. The excited carrier density was calculated from the laser wavelength, laser intensity, laser pulse width, and absorption coefficient (see supplementary material for experimental details).

It is common practice to normalize transient PL curves to the initial $(\mathrm{t} \approx 0)$ data point in order to compare multiple measurements. However, this approach can be sensitive to the very rapid dynamics that occur during and within the first few ns following the excitation pulse. This is illustrated in Fig. 2(a) using a simulated example. Normalizing the PL decay curves with different starting points [points 1-3 in Fig. 2(a), which are separated by only $200 \mathrm{ps}$ ] leads to a vertical shift in the normalized results [Fig. 2(b)]. While this may not

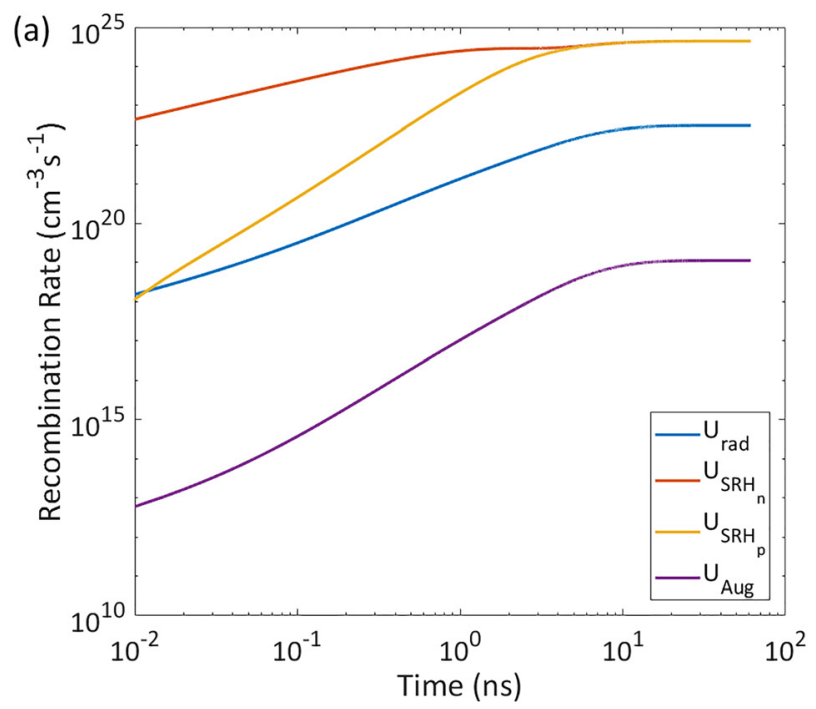

(b)

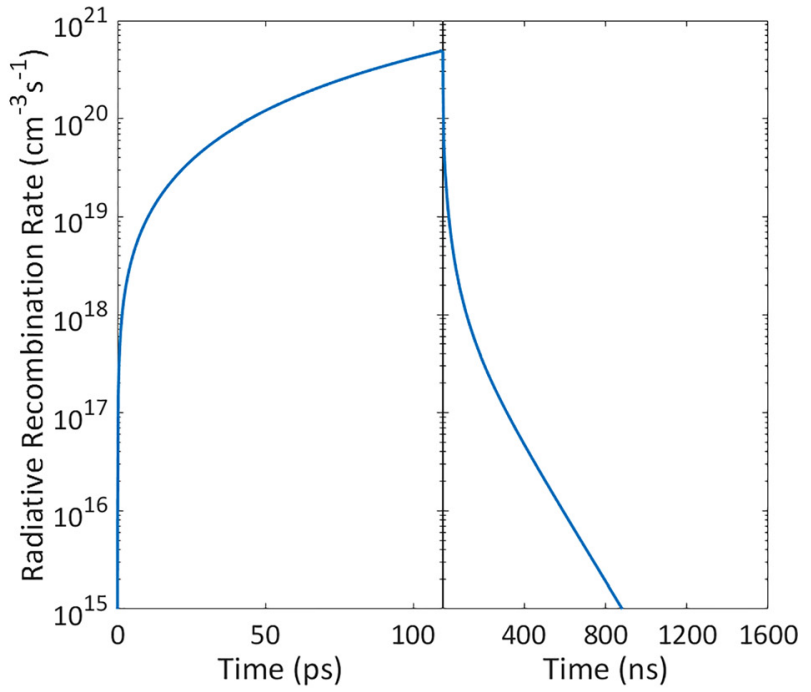

FIG. 1. (a) Modelled recombination rates as a function of time after turning on a constant illumination at $\mathrm{t}=0$ until the steady-state condition is reached. (b) Modelled radiative recombination rate as a function of time during one period of laser pulse (110 ps pulse width and $1600 \mathrm{~ns}$ repetition rate) in the transient PL measurement. Note that the time scales on the left hand side and right hand side of (b) are different. 
(a)

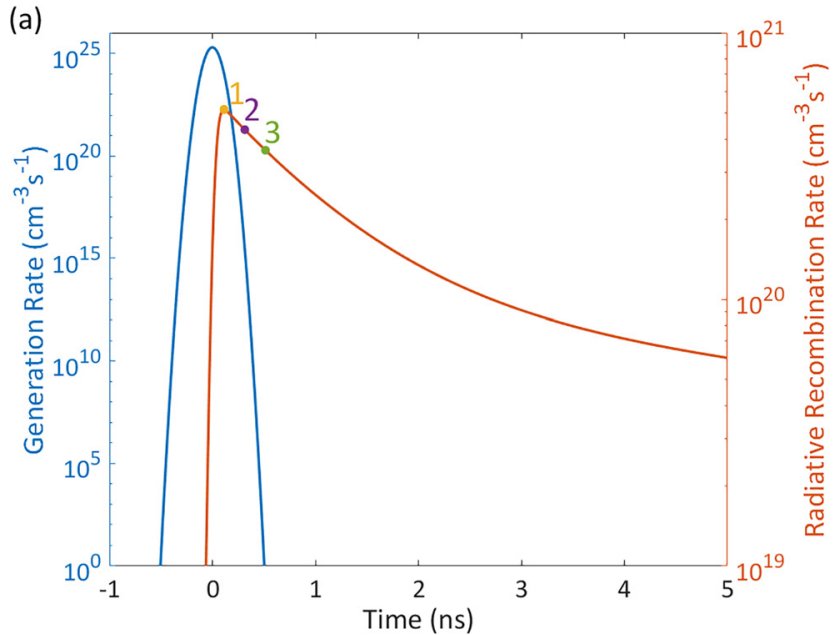

(b)

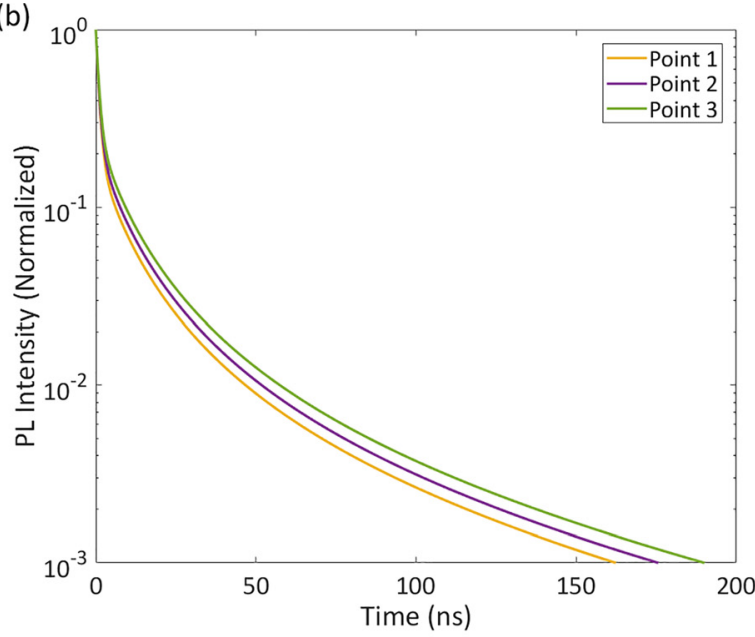

FIG. 2. (a) Simulated generation rate and transient radiative recombination rate during pulsed excitation. (b) The transient radiative recombination rate normalized at different initial data points 1-3 shown in (a), where these points are separated by $200 \mathrm{ps}$.

be significant when comparing relative decay rates between samples, it is a potential source of error when attempting to fit a numerical model to multiple decay curves measured under different conditions, as in this work. Thus, to minimize the uncertainty caused by normalization, here we instead normalize both the experimental and modelled transient PL curves at a later time of $\mathrm{t}=50 \mathrm{~ns}$ where the decay curves are less steep, and there is no possibility of contribution from the excitation pulse.

Before attempting to fit the recombination model to the experimental data, we first investigate the sensitivity of the steady-state and transient PL measurements to the trap parameters of interest using published parameter values as inputs to the model. For this part, we consider only $\mathrm{CH}_{3} \mathrm{NH}_{3} \mathrm{PbI}_{3}$, as published experimental data on the trap properties in multi-cation perovskite compositions are limited.

Table II summarizes the key material parameters of $\mathrm{CH}_{3} \mathrm{NH}_{3} \mathrm{PbI}_{3}$ perovskite films from several different publications. 5,8,10,13,14,21,22 Significant variations ( $>$ two orders of magnitude) are noted in the published values of radiative coefficient $(B)$, trap density $\left(N_{t}\right)$, and electron capture crosssection $\left(\left\langle c_{n}\right\rangle\right)$. Many different values have also been reported for trap energy levels $\left(E_{t}\right)$, including both shallow hole and shallow electron traps and deep (mid-gap) traps. This wide

TABLE II. Parameters of $\mathrm{CH}_{3} \mathrm{NH}_{3} \mathrm{PbI}_{3}$ from literatures.

\begin{tabular}{lc}
\hline \hline Parameters & Values \\
\hline$B\left(\mathrm{~cm}^{3} \mathrm{~s}^{-1}\right)$ & $1.3 \times 10^{-10}\left(\right.$ Ref. 5), $5.9 \times 10^{-13}$ (Ref. 13) \\
$N_{t}\left(\mathrm{~cm}^{-3}\right)$ & $2.5 \times 10^{16}\left(\right.$ Ref. 5), $7.44 \times 10^{16}$ (Ref. 14), \\
& $1.04 \times 10^{17}($ Ref. 14) \\
$E_{t}(\mathrm{eV})$ & $E_{c}-E_{t} \quad 0.62,0.75,0.76,^{8} 0.24,0.66^{10}$ \\
& $E_{t}-E_{v} \quad 0.129,0.024,0.16,0.459,0.508^{21}$ \\
$\left\langle c_{n}\right\rangle\left(\mathrm{cm}^{3} \mathrm{~s}^{-1}\right)$ & $2 \times 10^{-10}\left(\right.$ Ref. 5), $9.1 \times 10^{-13}($ Ref. 13) \\
$\left\langle e_{n}\right\rangle\left(\mathrm{cm}^{3} \mathrm{~s}^{-1}\right)$ & $8 \times 10^{-12}\left(\right.$ Ref. 5), 3.42 $\times 10^{-12}($ Ref. 13) \\
$\Gamma\left(\mathrm{cm}^{6} \mathrm{~s}^{-1}\right)$ & $1.08 \times 10^{-31}($ Ref. 13) \\
Doping & $N_{A}=4.0 \times 10^{16}\left(\right.$ Ref. 22), $N_{D}=2.8 \times 10^{17}$ (Ref. 22) \\
density $\left(\mathrm{cm}^{-3}\right)$ & \\
\hline \hline
\end{tabular}

variation may indicate either wide sample-to-sample variation or large experimental uncertainty (or both). The trap energy levels may also be influenced by whether the perovskite film is p-type or n-type, which has been found to vary depending on fabrication processes. ${ }^{22}$

Using as input parameters representative values from Table S1 in the supplementary material, Fig. 3 shows simulated PL intensity vs. excitation power curves [Figs. 3(a), 3(c), and 3(e)] and simulated transient PL curves [Figs. 3(b), 3(d), and 3(f)] for varying trap densities [Figs. 3(a) and 3(b)], trap energy levels [Figs. 3(c) and 3(d)], and electron capture coefficients [Figs. 3(e) and 3(f)]. The range of generation rates in the excitation-dependent steady-state PL simulation and the excitation level used in the transient PL simulation $\left(\sim 1 \times 10^{24} \mathrm{~cm}^{-3} \mathrm{~s}^{-1}\right)$ were chosen to approximate our experimental conditions. Note that the decision to normalize the transient PL curves at a time of $50 \mathrm{~ns}$ results in apparent trends that may appear different from the standard approach of normalizing to the initial intensity. This does not affect the following parameter fitting procedure since the experimental and simulated curves are normalized in the same way.

As expected, Fig. 3(a) shows that both the shape and magnitude of the steady-state PL intensity curves (radiative recombination rate) are relatively sensitive to changes in the trap density under the simulated conditions. The corresponding transient PL curves in Fig. 3(b) also exhibit a dependence on these parameters, but this dependence weakens at higher trap densities. This suggests that intensity-dependent steady-state PL measurements may be more accurate for extracting trap density when the density is high. In contrast, the steady-state PL curves plotted in Fig. 3(c) show only a very weak dependence on the trap energy level, whereas the transient PL curves have a much stronger dependence. This may be a consequence of the relatively high generation rates simulated for the steadystate case: when the density of photogenerated carriers is much higher than the trap density, the trap occupation rate becomes saturated. The stronger dependence in Fig. 3(d) results from different rates of electron capture and 
(a)

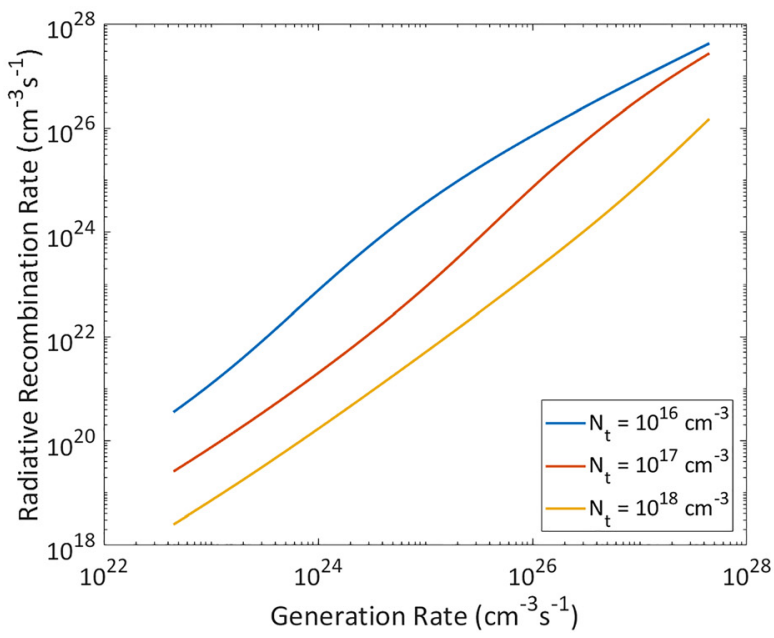

(c)

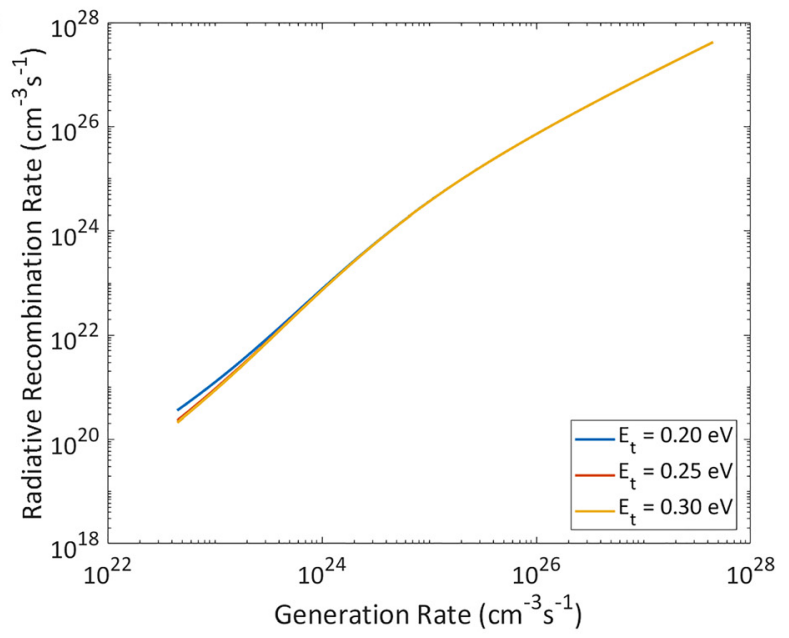

(e)

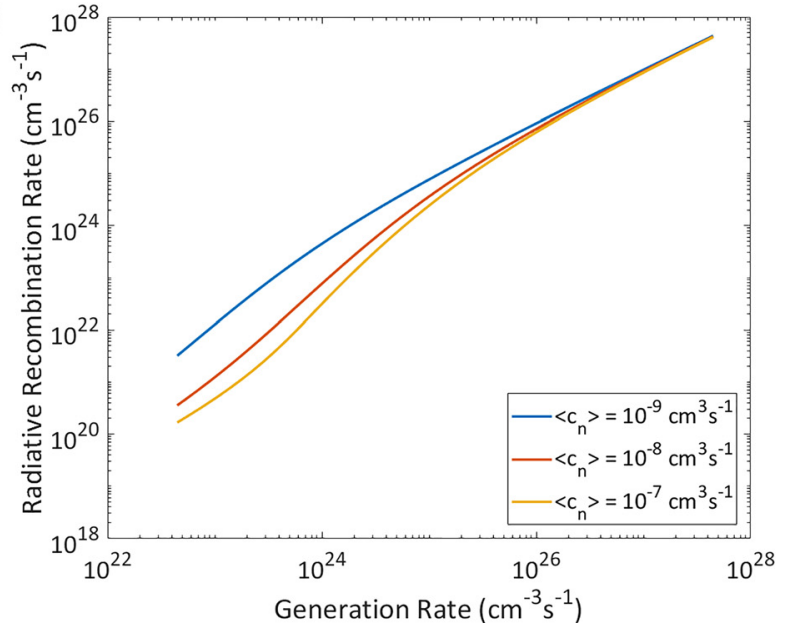

(b)

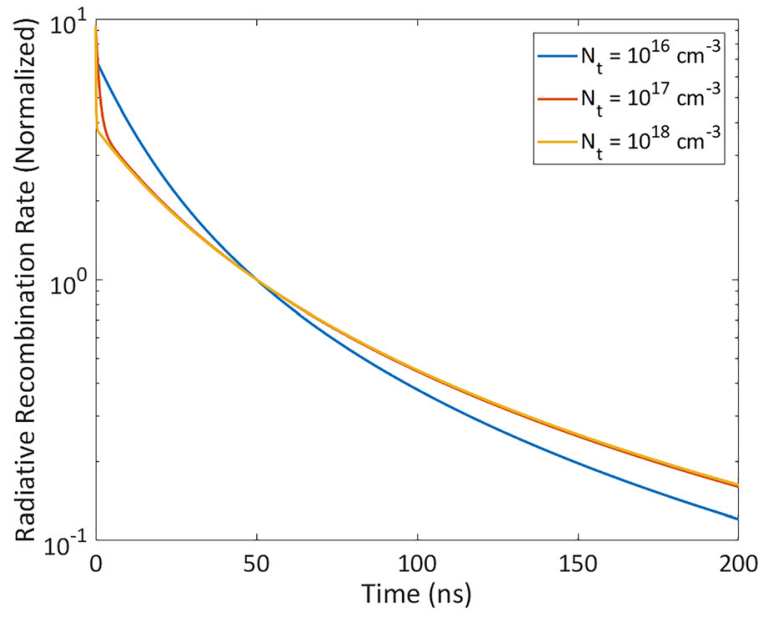

(d)

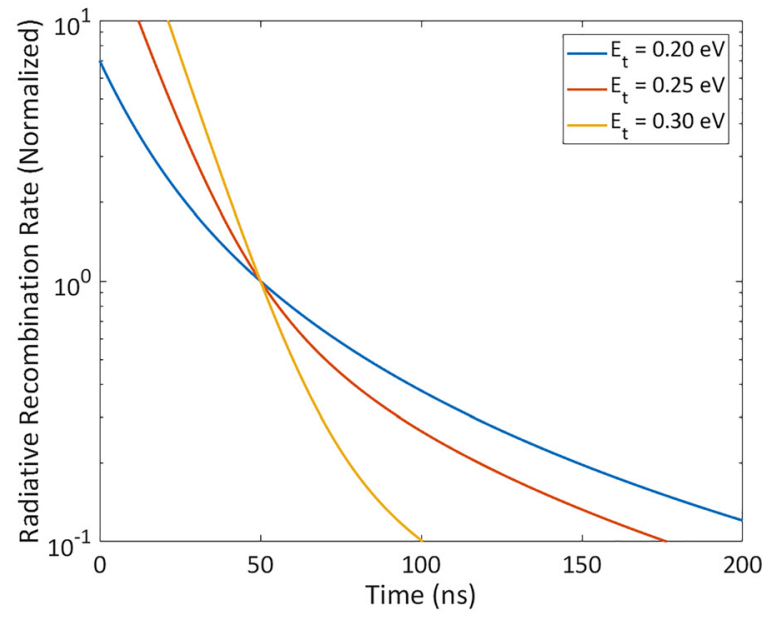

(f)

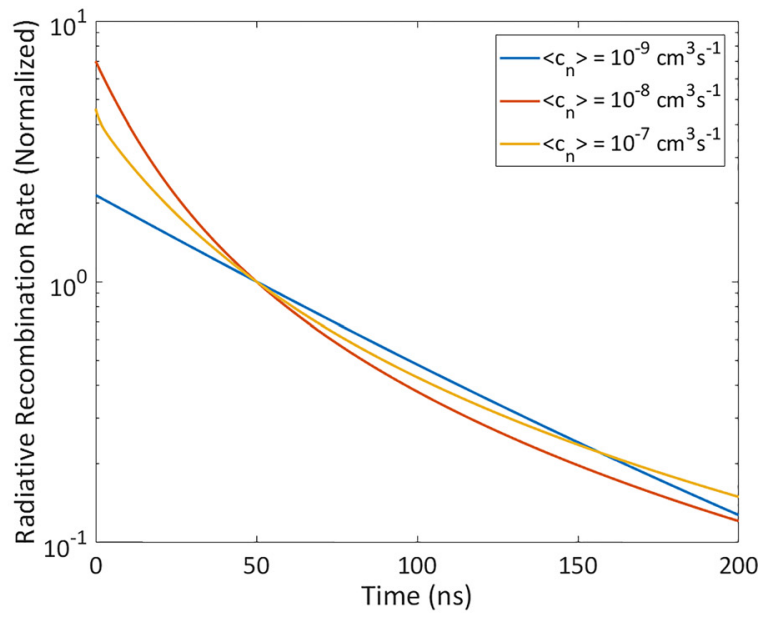

FIG. 3. The simulated excitation-dependent steady-state PL measurement with different (a) trap densities $N_{t}$, (c) trap energy levels $E_{t}$, and (e) capture coefficients of the electron $\left\langle c_{n}\right\rangle$; the simulated excitation-dependent transient PL measurement with different (b) trap densities $N_{t}$, (d) trap energy levels $E_{t}$, and (f) capture coefficients of the electron $\left\langle c_{n}\right\rangle$. The input values are listed in Table S1.

emission at the low excitation level, which are sensitive to the trap energy level. Figures 3(e) and 3(f) exhibit the behavior between the previous two cases: the steady-state PL curves are relatively sensitive to the electron capture coefficient at low generation rates but converge as the generation rate is increased. The transient PL curves in Fig. 3(f) show a transition from a single exponential (radiative recombination-dominated) decay when the capture coefficient is smaller than the emission coefficient (blue curve) to a non-exponential decay at higher capture coefficient values.

The results in Fig. 3 show that at least in some cases, transient PL measurements are relatively insensitive to $N_{t}$ 

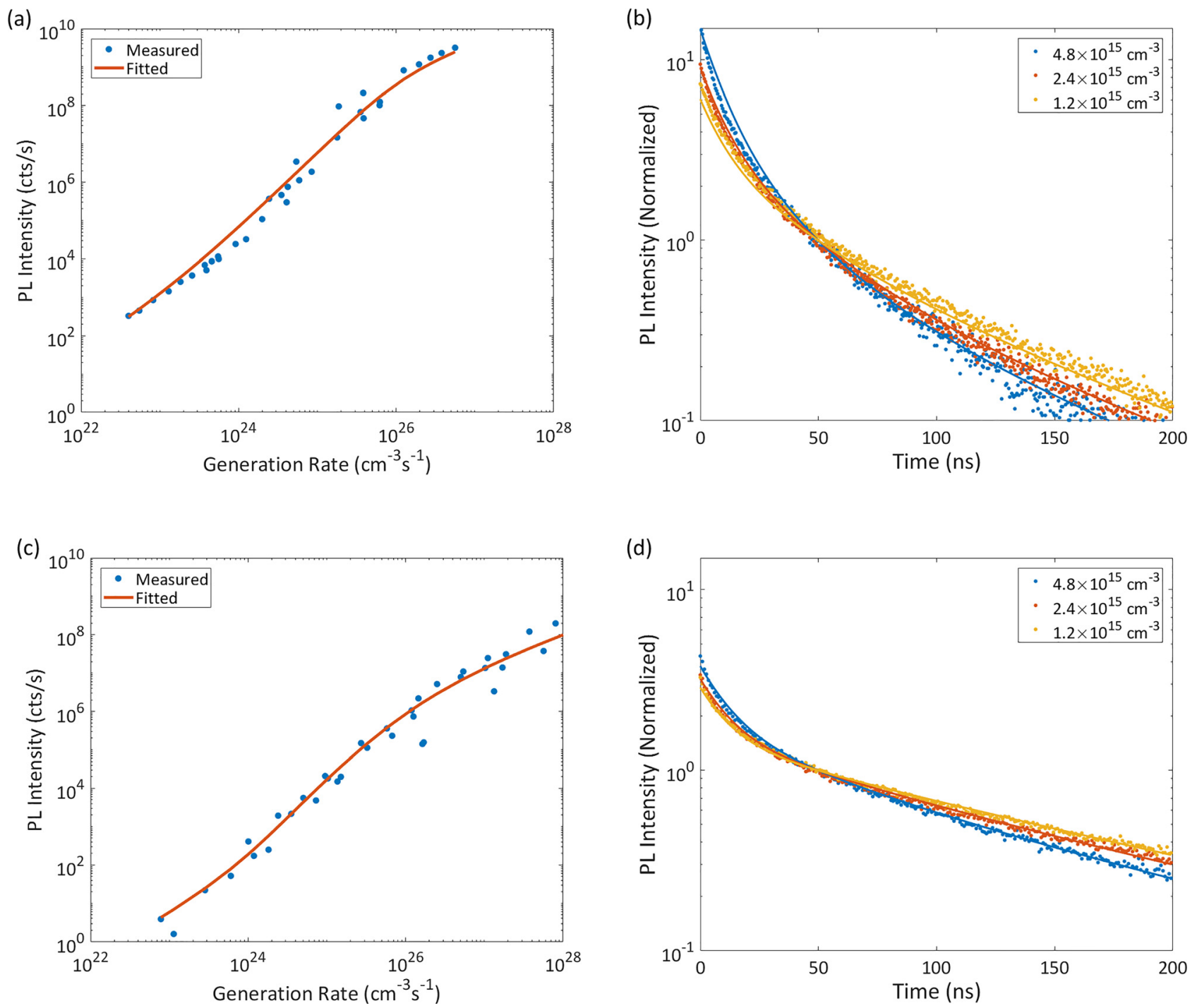

FIG. 4. Measured (blue dots) and modelled (red line) steady-state PL peak intensities as a function of the carrier generation rate on the (a) MAPbI 3 perovskite film and (c) quadruple-cation perovskite film (the laser wavelength is $532 \mathrm{~nm}$ ). Measured (dots) and modelled (lines) transient PLs with different excitation levels on the (b) $\mathrm{MAPbI}_{3}$ perovskite film and (d) quadruple-cation perovskite film (508 nm pulsed laser and $625 \mathrm{kHz}$ repetition rate).

[Fig. 3(b)], while excitation-dependent PL intensity shows little dependence on $E_{t}$ [Fig. 3(c)] within the experimental conditions simulated. Thus, attempting to extract values of trap properties using only one of these PL measurement techniques would result in large uncertainties. However, fitting a single recombination model to both sets of experimental data simultaneously can significantly improve the accuracy of the fitted trap parameters, as we show below. We further improve the accuracy of the parameter extraction by including in the fitting routine three sets of transient PL spectra for each sample, measured at different excitation levels.

\section{EXPERIMENTAL RESULTS}

Two perovskite compositions were used in this experiment: the widely studied $\mathrm{MAPbI}_{3}$ and the more recently developed quadruple-cation/mixed halide perovskite $\mathrm{Cs}_{0.07} \mathrm{Rb}_{0.03}\left(\mathrm{FA}_{0.85} \mathrm{MA}_{0.15}\right)_{0.9} \mathrm{~Pb}\left(\mathrm{I}_{0.85} \mathrm{Br}_{0.15}\right)_{3}$. Both compositions were deposited as planar films on a glass substrate as described in the supplementary material. Both films were coated with PMMA to passivate surface defects ${ }^{23,24}$ and to provide some protection from ambient humidity. To further protect samples, the measurements were performed in a nitrogen environment. Example J-V curves of perovskite solar cells produced by the same film fabrication processes are shown in Figs. S4 and S5 in the supplementary material.

Steady-state and transient PL measurements were carried out in a confocal microscope during a single measurement session. Further details of the experimental set-up and laser sources are provided in the supplementary material. Steady-state PL spectra were collected first by varying the laser intensity over several orders of magnitude, corresponding to generation rates from $10^{23}$ to $10^{28} \mathrm{~cm}^{-3} \mathrm{~s}^{-1}$ (the calculation of the generation rate is shown in the supplementary material). This range of generation rates spans operating regions previously identified as being dominated by monomolecular and bimolecular recombination ${ }^{25,26}$ and at the highest intensities starts to enter the Auger recombination regime. The PL peak intensities are plotted in Figs. 4(a) and 4(c) as a function of the generation rate. Next, time-resolved 
PL spectra were measured at the same location on the sample at three different excitation levels [Figs. 4(b) and 4(d)].

To confirm the sample stability during the experiment, films were exposed to the maximum laser intensity used in the steady-state PL measurements for $10 \mathrm{~min}$, and the PL emission was monitored. Samples were tested in two locations: first, on an area of the sample that had already been measured, and second, on a fresh (non-exposed) area. No significant change in the PL intensity was observed in either case (Fig. S3 in the supplementary material).

From the PL spectra of the two samples, we estimate a bandgap of $1.6 \mathrm{eV}$ for both (Fig. S2 in the supplementary material). The absorption coefficients of $\mathrm{MAPbI}_{3}$ and quadruple-cation perovskite films were taken from published values to be $1.5 \times 10^{4} \mathrm{~cm}^{-1}$ (Ref. 27) and $1.2 \times 10^{5} \mathrm{~cm}^{-1}$ (Ref. 28), respectively, at a wavelength of approximately $500 \mathrm{~nm}$ for the purpose of calculating generation rates within the films.

In the recombination model, seven parameters $\left(B, N_{t}\right.$, $E_{t},\left\langle c_{n}\right\rangle,\left\langle e_{n}\right\rangle, \Gamma$, and $\left.N_{A}\right)$ are unknown. Here, an electron trapping nature is assumed as in Ref. 5 for p-type perovskite materials, corresponding to a trap energy level between the mid-gap and the conduction band. We also assume that the Auger coefficients are equal $\left(\Gamma_{n}=\Gamma_{p}=\Gamma\right)$ to simplify the fitting, as has also been assumed in previous works. ${ }^{13}$

The recombination model was fitted simultaneously to the four sets of experimental data (one set of steady state PL vs excitation level data and three sets of transient PL data). A single, combined value of normalized root mean square error (NRMSE) was defined (see supplementary material for details) and minimized using a nonlinear optimization routine. The process was repeated several times with different starting conditions to ensure that a stable, global minimum had been found.

The fitted numerical results are plotted in Fig. 4 as solid curves, and the fitted parameters are listed in Table III.

When comparing the experimental data to the numerical model, there are two distinct sources of error that contribute to the NRMSE. The first is noise in the raw experimental data that can be seen clearly in Fig. 4. The second source of error is related to how accurately the numerical model describes the recombination kinetics. In the discussion below, we refer to these as the experimental error and fitting error, respectively. The presence of experimental error means that even if the model captures all of the relevant physical processes, the total NRMSE will remain non-zero.
Thus, it is desirable to separate the two error contributions. To approximate this, we first generate a smoothed set of experimental data by applying a moving average function. The experimental uncertainty is then defined as the NRMSE between the smoothed moving average curve and the raw (noisy) data, while the fitting error is defined as the NRMSE between the model and the smoothed experimental data. With this definition, we calculate experimental NRMSE values of 0.1 for both the $\mathrm{MAPbI}_{3}$ and quadruple-cation perovskite films. Using this estimated experimental error as an upper error limit, we define the uncertainty of individual fitted parameters as the range of parameter values within which the fitted NRMSE remains smaller than the experimental NRMSE. Although this is somewhat arbitrary choice, it provides a consistent definition for assigning an uncertainty to the fitted parameters. These uncertainties are expressed as upper and lower bounds on each parameter in Table III.

As can be seen in Table III, the above approach for defining uncertainties results in undefined lower bounds on the radiative recombination coefficient $B$ and Auger recombination coefficient $\Gamma$. To investigate this further, we next consider the sensitivities of the fitted parameters to the two different types of experimental measurements: excitationdependent steady-state PL and transient PL.

The blue curves in each sub figure of Fig. 5 show the individual fitted NRMSE contributions associated with the steady-state PL data (solid curves), the transient PL data (dashed curves), and the total (combined) NRMSE contributions (solid curve with circles) for the $\mathrm{MAPbI}_{3}$ sample as a function of a specific fitting parameter. The equivalent data are also shown for the quadruple-cation perovskite sample (red curves). Consider first the blue curves in Fig. 5(a). In this case, the quality of the fit to both types of PL measurements is seen to be insensitive to the value of the radiative recombination coefficient $B$ except in cases of very large values of $B$. Thus, it is only possible to identify an upper bound for the value of $B$ but no lower bound. A similar situation is seen in Fig. 5(f) for the Auger recombination coefficient $\Gamma$.

The remaining plots in Fig. 5 demonstrate that the fitted parameters are generally more sensitive to one type of experimental measurement than another, illustrated by a narrow, well-defined minimum. This observation further justifies the value of using both steady-state PL and transient PL data to fit the recombination model. This is particularly obvious in the case of the electron and hole capture coefficients [Figs. 5(d) and 5(e)], where the transient PL data show a much

TABLE III. Fitted results and uncertainty bounds of each parameter.

\begin{tabular}{|c|c|c|c|c|c|c|}
\hline \multirow[b]{2}{*}{ Fitted parameters } & \multicolumn{3}{|c|}{$\mathrm{CH}_{3} \mathrm{NH}_{3} \mathrm{PbI}_{3}$} & \multicolumn{3}{|c|}{$\mathrm{Cs}_{0.07} \mathrm{Rb}_{0.03} \mathrm{FA}_{0.765} \mathrm{MA}_{0.135} \mathrm{PbI}_{2.55} \mathrm{Br}_{0.45}$} \\
\hline & Fitted value & Lower bound & Upper bound & Fitted value & Lower bound & Upper bound \\
\hline$B\left(\mathrm{~cm}^{3} \mathrm{~s}^{-1}\right)$ & $7 \times 10^{-12}$ & $\mathrm{~N} / \mathrm{A}$ & $7.8 \times 10^{-11}$ & $3 \times 10^{-12}$ & $\mathrm{~N} / \mathrm{A}$ & $3.0 \times 10^{-11}$ \\
\hline$N_{t}\left(\mathrm{~cm}^{-3}\right)$ & $1 \times 10^{16}$ & $8.6 \times 10^{15}$ & $2.3 \times 10^{16}$ & $8 \times 10^{16}$ & $6.4 \times 10^{16}$ & $1.1 \times 10^{17}$ \\
\hline$E_{t}(\mathrm{eV})$ & 0.20 & 0.188 & 0.202 & 0.17 & 0.161 & 0.172 \\
\hline$\left\langle c_{n}\right\rangle\left(\mathrm{cm}^{3} \mathrm{~s}^{-1}\right)$ & $8 \times 10^{-9}$ & $6.6 \times 10^{-9}$ & $1.1 \times 10^{-8}$ & $1 \times 10^{-9}$ & $8.7 \times 10^{-10}$ & $1.2 \times 10^{-9}$ \\
\hline$\left\langle e_{n}\right\rangle\left(\mathrm{cm}^{3} \mathrm{~s}^{-1}\right)$ & $4 \times 10^{-8}$ & $3.8 \times 10^{-8}$ & $4.5 \times 10^{-8}$ & $4 \times 10^{-10}$ & $3.4 \times 10^{-10}$ & $3.9 \times 10^{-10}$ \\
\hline$\Gamma\left(\mathrm{cm}^{6} \mathrm{~s}^{-1}\right)$ & $1 \times 10^{-30}$ & $\mathrm{~N} / \mathrm{A}$ & $3.6 \times 10^{-29}$ & $8 \times 10^{-32}$ & $\mathrm{~N} / \mathrm{A}$ & $8.0 \times 10^{-31}$ \\
\hline$N_{A}\left(\mathrm{~cm}^{-3}\right)$ & $3 \times 10^{14}$ & $2.5 \times 10^{14}$ & $3.3 \times 10^{14}$ & $2 \times 10^{16}$ & $1.7 \times 10^{16}$ & $1.9 \times 10^{16}$ \\
\hline
\end{tabular}



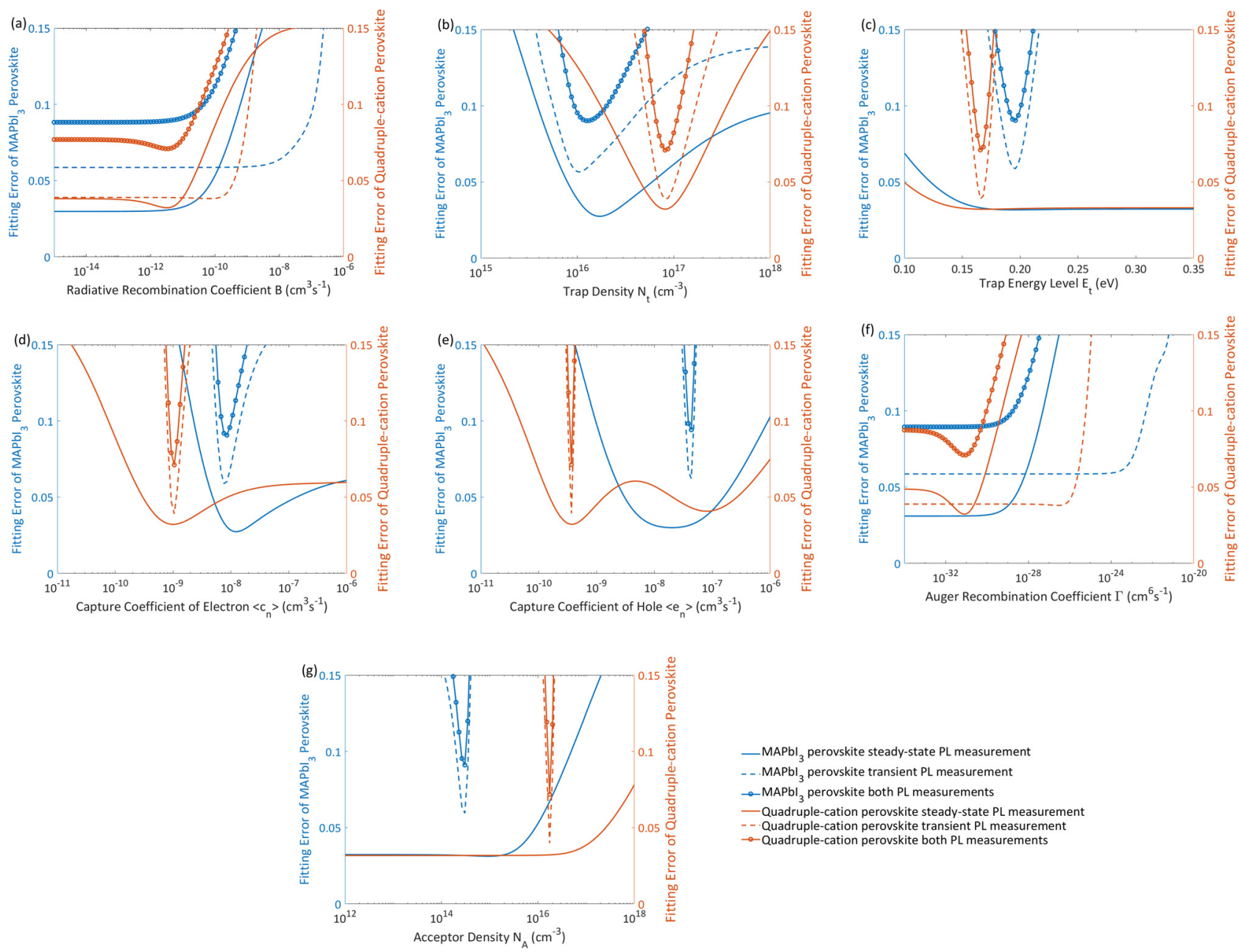

FIG. 5. The fitting error (NRMSE) of steady-state PL measurements (solid lines), transient PL measurements (dashed lines), and combined experiments (solid line with marker) on the $\mathrm{MAPbI}_{3}$ perovskite film (blue curves) and the quadruple-cation perovskite film (red curves) when sweeping (a) radiative recombination coefficient $B$, (b) trap density $N_{t}$, (c) trap energy level $E_{t}$, (d) capture coefficient of the electron $c_{n}$, (e) capture coefficient of the hole $e_{n}$, (f) Auger recombination coefficient $\Gamma$, and (g) acceptor density $N_{A}$.

higher sensitivity to these parameters than the steady-state data. This is also the case for the trap energy level [Fig. 5(c)]. Note also that there is a local minimum in the NRMSE when sweeping $\left\langle e_{n}\right\rangle$ which illustrates the importance of finding the global minimum during the fitting procedure [Fig. 5(e)]. This analysis demonstrates that it is indeed possible to extract constrained parameter values for all of the key trap parameters by fitting a single general recombination model to both steady-state and transient PL measurements.

The parameter values extracted for $\mathrm{MAPbI}_{3}$ are consistent with those from the literature in Table II. The value of $E_{t}$ indicates the existence of relatively shallow traps close to the conduction band level. The result that the capture coefficient of electrons is larger than that of holes also agrees with the assumption of electronic traps.

\section{DISCUSSION}

Having extracted the trap parameters and estimated the uncertainties, we can now make a comparison of the two perovskite compositions. As reported by a number of groups, quadruple-cation perovskite solar cells can achieve higher open-circuit voltage than $\mathrm{MAPbI}_{3}$-based cells. The open- circuit voltage of a solar cell depends strongly on the relative rates of radiative and non-radiative recombination in the active layer. In order to maximize $V_{O C}$, the non-radiative recombination-SRH recombination and Auger recombination-should be minimized. Under standard one-sun illumination, recombination in perovskite films is dominated by SRH recombination. Therefore, $N_{t}, E_{t},\left\langle c_{n}\right\rangle$, and $\left\langle e_{n}\right\rangle$ are the most important parameters for determining the recombination properties. Considering the fitted results of these four parameters in Table III, the trap densities of the two samples are similar; but the trap energy level of the $\mathrm{MAPbI}_{3}$ film is deeper than that of the quadruple-cation perovskite film, which means that the fraction of traps working as recombination centers is higher in the $\mathrm{MAPbI}_{3}$ film. The most significant difference between the two samples is in the capture coefficient of holes. The extracted capture cross section of the quadruple-cation perovskite is up to two orders of magnitude smaller than that of the $\mathrm{MAPbI}_{3}$ film. This suggests that it is more difficult for carriers to be trapped in the quadruplecation film, resulting in a significantly lower non-radiative recombination rate. While this single observation from one sample of each material is insufficient to draw firm conclusions, it suggests that the improved performance of mixed 
cation perovskites may result from less active trap states rather than from a lower density of traps. If confirmed by further studies, this finding could provide new insights into the apparent defect-tolerance of perovskite materials.

\section{CONCLUSION}

This work has demonstrated an improved method for extracting recombination parameters by fitting a generalized model for recombination kinetics to excitation-dependent steady-state and transient PL measurements. Sensitivity analysis shows that fitting a single model to the two different PL measurements provides improved accuracy in fitting multiple trap parameters compared to fitting to a single type of measurement. Consequently, we extracted recombination coefficients and trap parameters and the associated uncertainties of these fitted parameters.

The quality of the fits obtained with a single trap state suggests that either non-radiative recombination is dominated by a single trap species or the combined contribution of active traps can be approximated by a single set of "effective" trap parameters. Further studies are required to resolve which of these interpretations is correct. Nevertheless, the analysis techniques presented here provide a simple, non-contact method to rapidly characterize the key trap characteristics of perovskite films. Since the method is based on a general physical recombination model, it could, in principle, be readily applied to other semiconductor materials beyond metal-halide perovskites, providing new insight into their recombination kinetics.

\section{SUPPLEMENTARY MATERIAL}

See supplementary material for the sample fabrication processes, experimental methods, and the calculations of the generation rate and NRMSE.

\section{ACKNOWLEDGMENTS}

The authors thank Dr. The Duong and Dr. Jun Peng for providing the fabrication processes of perovskite films and the J-V curves of the perovskite solar cells. They also thank Australian Renewable Energy Agency (ARENA) for funding.
${ }^{1}$ A. Kojima, K. Teshima, Y. Shirai, and T. Miyasaka, J. Am. Chem. Soc. 131, 6050-6051 (2009).

${ }^{2}$ See https://www.nrel.gov/pv/assets/images/efficiency-chart.png for Best Research-Cell Efficiencies (NREL, 2018).

${ }^{3}$ Y. Li, B. Ding, Q.-Q. Chu, G.-J. Yang, M. Wang, C.-X. Li, and C.-J. Li, Sci. Rep. 7, 46141 (2017).

${ }^{4}$ W. Tress, Adv. Energy Mater. 7, 1602358 (2017).

${ }^{5}$ S. D. Stranks, V. M. Burlakov, T. Leijtens, J. M. Ball, A. Goriely, and H. J. Snaith, Phys. Rev. Appl. 2, 034007 (2014).

${ }^{6}$ T. S. Sherkar, C. Momblona, L. Gil-Escrig, J. Ávila, M. Sessolo, H. J. Bolink, and L. J. A. Koster, ACS Energy Lett. 2, 1214-1222 (2017).

${ }^{7}$ G. Gordillo, C. A. Otálora, and M. A. Reinoso, J. Appl. Phys. 122, 075304 (2017).

${ }^{8}$ S. Heo, G. Seo, Y. Lee, D. Lee, M. Seol, J. Lee, J.-B. Park, K. Kim, D.-J. Yun, Y. S. Kim, J. K. Shin, T. K. Ahn, and M. K. Nazeeruddin, Energy Environ. Sci. 10, 1128-1133 (2017).

${ }^{9}$ G. Landi, H. C. Neitzert, C. Barone, C. Mauro, F. Lang, S. Albrecht, B. Rech, and S. Pagano, Adv. Sci. 4, 1700183 (2017).

${ }^{10}$ M. Samiee, S. Konduri, B. Ganapathy, R. Kottokkaran, H. A. Abbas, A. Kitahara, P. Joshi, L. Zhang, M. Noack, and V. Dalal, Appl. Phys. Lett. $\mathbf{1 0 5}, 153502$ (2014).

${ }^{11}$ J. S. Manser and P. V. Kamat, Nat. Photonics 8, 737-743 (2014).

${ }^{12}$ C. Wehrenfenning, M. Liu, H. J. Snaith, M. B. Johnston, and L. M. Herz, Energy Environ. Sci. 7, 2269-2275 (2014).

${ }^{13}$ L. H. Manger, M. B. Rowley, Y. Fu, A. K. Foote, M. T. Rea, S. L. Wood, S. Jin, J. C. Wright, and R. H. Goldsmith, J. Phys. Chem. C 121, 1062-1071 (2017).

${ }^{14}$ X. Wen, Y. Feng, S. Huang, F. Huang, Y.-B. Cheng, M. Green, and A. Ho-Baillie, J. Mater. Chem. C 4, 793-800 (2016).

${ }^{15}$ W. Shockley and W. T. Read, Jr., Phys. Rev. 87, 835 (1952).

${ }^{16}$ R. N. Hall, Phys. Rev. 83, 228 (1951).

${ }^{17}$ R. N. Hall, Phys. Rev. 87, 387 (1952).

${ }^{18}$ M. A. Green, Solar Cells: Operating Principles, Technology, and System Applications (University of New South Wales, 1986), pp. 50-52.

${ }^{19}$ P. Auger, C. R. Acad. Sci. 177, 169-171 (1923).

${ }^{20}$ G. Giorgi, J.-I. Fujisawa, H. Segawa, and K. Yamashita, J. Phys. Chem. Lett. 4, 4213-4216 (2013)

${ }^{21}$ G. Gordillo, C. A. Otálora, and A. A. Ramirez, Phys. Chem. Chem. Phys. 18, 32862-32867 (2016).

${ }^{22}$ T. Zhao, W. Shi, J. Xi, D. Wang, and Z. Shuai, Sci. Rep. 6, 19968 (2016).

${ }^{23}$ J. Peng, Y. Wu, W. Ye, D. A. Jacobs, H. Shen, X. Fu, Y. Wan, T. Duong, N. Wu, C. Barugkin, H. T. Nguyen, D. Zhong, J. Li, T. Lu, Y. Liu, M. N. Lockrey, K. J. Weber, K. R. Catchpole, and T. P. White, Energy Environ. Sci. 10, 1792-1800 (2017).

${ }^{24}$ F. Wang, A. Shimazaki, F. Yang, K. Kanahashi, K. Matsuki, Y. Miyauchi, T. Takenobu, A. Wakamiya, Y. Murate, and K. Matsuda, J. Phys. Chem. C 121, 1562-1568 (2017).

${ }^{25}$ M. Saba, M. Cadelano, D. Marongiu, F. Chen, V. Sarritzu, N. Sestu, C. Figus, M. Aresti, R. Piras, A. G. Lehmann, C. Cannas, A. Musinu, F. Quochi, A. Mura, and G. Bongiovanni, Nat. Commun. 5, 5049 (2014).

${ }^{26}$ C. M. Sutter-Fella, Y. Li, M. Amani, J. W. Ager III, F. M. Toma, E. Yablonovitch, I. D. Sharp, and A. Javey, Nano Lett. 16, 800-806 (2016).

${ }^{27}$ N.-G. Park, Mater. Today 18, 65-72 (2015).

${ }^{28}$ Y. Sun, J. Peng, Y. Chen, Y. Yao, and Z. Liang, Sci. Rep. 7, 46193 (2017). 